\title{
Revisión conjunta de fuentes primordiales para autoabastecimiento energético urbano e incidencia solar como principal fuente, en contexto de ciudad ecuatorial-andina
}

\author{
Esteban Zalamea-León ${ }^{1 *}$, Antonio Barragán-Escandón ${ }^{2}$ \\ Universidad de Cuenca \\ ${ }^{2}$ Universidad Politécnica Salesiana \\ *Autor para correspondencia / Corresponding autor: esteban.zalamea@ucuenca.edu.ec
}

\section{Review of primary sources for urban energy self-supply and solar incidence as the main source, in the context of the Equatorial-Andean city}

\begin{abstract}
This work reviews individual studies determining different energy technological possibilities and capabilities, in order to figure out their joint potential of urban energy self-supply, with internal resources. This study has been done in the context of the Andean equatorial city of Cuenca, Ecuador, as a representative case. Initially, the existing alternatives are described, selecting six options through an expert survey. Then individual estimations of energy capability of the defined resource and, from that, a joint potential has been found. When comparing the capabilities and against real urban consumption, has been establishing that solar energy is by far the source with the greatest potential and with better adaptability from qualitative and quantitative aspects. Besides It has been found that with the analyzed technologies, together, $14 \%$ of the urban consumption of 2015 as base year can be supplied. Nevertheless, it is observed that as a consequence of the PV potential, this potential is increased to $39 \%$, but for this option could be useful, a conversion of consumptions from fuels to electricity is required. In consequence, then is presented the PV potential of typologies that have been determined in buildings of different types in the city. From this it is concluded that the existing Andean equatorial climatic conditions in Cuenca are highly advantageous to reach maximum urban and building energy standards with solar energy, due to the reduced and stable temporary demands, in addition to good and constant presence of irradiation.
\end{abstract}

Editado por / Edited by: Eva O.L. Lantsoght

Recibido / Received: $06 / 11 / 2019$

Aceptado /

Accepted:

02/03/2020

Publicado en línea

/ Published online:

01/05/2020

Keywords: Renewable energies, Urban energy metabolism, energy on buildings, solar energy.

\section{Resumen}

Este trabajo realiza la revisión conjunta de una serie de investigaciones que determinan individualmente distintas posibilidades tecnológicas y sus capacidades para alcanzar un potencial de autoaprovisionamiento energético desde recursos urbanos. Este estudio se realiza en la ciudad ecuatorial andina de Cuenca, Ecuador, como caso representativo. Inicialmente se describen las alternativas tecnológicas existentes, considerando seis 
de estas en este estudio. Al compararse la disponibilidad de los recursos energéticos y los consumos urbanos detectados, se establece que la energía solar es por lejos la fuente con mayor potencial y con mejor adaptabilidad desde aspectos cualitativos y cuantitativos. Se determina que con las tecnologías analizadas, se puede abastecer sobre el $14 \%$ de los consumos urbanos del año base 2015. No obstante, se observa que como consecuencia del potencial PV, este potencial se incrementa hasta un 39\%, llegando incluso a tener excedentes eléctricos en momentos puntuales, lo que sugiere realizar una conversión de consumos desde combustibles a eléctricos, lo que demuestra un potencial superior para esta tecnología. Se concluye que las condiciones climáticas ecuatoriales andinas existentes en Cuenca resultan altamente ventajosas para alcanzar máximos estándares energéticos urbanos y en edificios, por las reducidas y estables demandas temporales, además debido al buen y constante potencial de energía solar.

Palabras clave: Energías renovables, Metabolismo energético urbano, edificación, energía solar.

\section{INTRODUCCIÓN}

Los centros urbanos son foco del problema climático del planeta, pero también existe potencial relativo para alcanzar resiliencia y equilibrio ambiental [1]. Para ello es estratégico la implementación de medidas de eficiencia energética sumado a un autoaprovisionamiento energético desde recursos endógenos como alternativa idónea [2]. Varias opciones se han extendido con relativo éxito, sobre todo en países desarrollados [3] Este proceso debe expandirse a aquellos en vías de desarrollo [4-6].

Tal como se ha evidenciado en Ecuador y Latinoamérica [7,8] los problemas asociados con la energía resultan en problemas que pueden implicar crisis sociales e incluso de gobernabilidad. Ello implica la necesidad de impulsar alternativas limpias que puedan subsidiarse para descarbonizar los procesos sociales y económicos. Las evoluciones tecnológicas y la economía de escala son determinantes para alcanzar viabilidad económica [9-11]. La creación de fuentes trabajo locales también es una ventaja comparativa sustancial, así, las tecnologías renovables en microgeneración frente a las tradicionales implican entre seis y diez veces más plazas locales de mano de obra [12]. El sector de Energías Renovables (ER) muestra crecimiento mundial de más del 5\% al año, no observado en ningún otro sector productivo [12,13]. Más allá de ello, las fuentes no renovables e incluso fuentes renovables instaladas a gran escala en lugares agrestes, también implican impacto en ocupación y afección a la naturaleza, como lo describen en sus investigaciones Poggi, Firmino, \& Amado, [14]para el caso de hidroeléctricas con grandes represas, así como también el impacto establecido por el consumo de biomasa a gran escala en el estudio de Caldarelli \& Gilio, [16]. Entonces, la opción es promover un metabolismo urbano circular y en este ámbito las opciones existentes para lograr una máxima generación urbana, con lo cual se puede reducir además la importación de materiales o alimentos como también de energía [17,18].

Una de las principales barreras para limitar el autoabastecimiento energético de ciudades es la fluctuación de consumos y la intermitencia de las fuentes energéticas renovables como 
solar o eólica [19,20]. Pero en las condiciones climáticas de ciudades ecuatoriales andinas se observan características muy adecuadas para alcanzar un margen de autogeneración significativo, ya que el consumo energético es relativamente estable durante el año, así como también la intermitencia de fuentes que dependen del clima y de las estaciones, lo que es menor cuando se compara con ciudades más alejadas del Ecuador.

A continuación, se describen de manera simplificada varias metodologías y procesos que han permitido determinar la capacidad de autogeneración de forma. Se describen las posibilidades tecnológicas en el contexto de Cuenca, Ecuador, como ciudad intermedia representativa de la condición ecuatorial andina. Consideramos que esta condición puede ser representativa al localizarse a 2535 msnm y bajo condiciones climáticas de ciudades localizadas cerca de la línea ecuatorial a altura comprendida sobre los 2000 msnm y bajo los 3000 msnm considerando su similitud climática. Así en la Tabla 1 se recogen ciudades localizadas en los valles interandinos, cuyas temperaturas promedio están entre los $16,5^{\circ} \mathrm{C}$ y $13,0^{\circ} \mathrm{C}$; con máxima promedio de $22,8^{\circ}$ y mínimas promedias entre $11,3^{\circ} \mathrm{C}$ y $7,1^{\circ} \mathrm{C}$, con la excepción de Cajamarca (Perú) en cuyo caso el promedio mínimo es de $2,1^{\circ} \mathrm{C}$. Son temperaturas que se encuentran por debajo de los límites de confort térmico, no obstante, utilizando diseños pasivos en las edificaciones se pueden alcanzar los niveles de confort requeridos con un bajo o nulo aporte de energía adicional. Si bien se pueden tener horas en las que la temperatura se aleja de los límites adecuados de confort térmico, con el uso de una adecuada ventilación, captación solar pasiva y control de niveles de infiltración se pueden alcanzar temperaturas internas adecuadas. Como se puede observar en dicha tabla, Cuenca posee temperaturas intermedias entre las ciudades descritas, por lo que consideramos que se trata de un caso representativo.

Tabla 1: Temperaturas promedio máxima y mínima promedio de ciudades entre los 2000 y 3000 msnm, próximas a la línea ecuatorial (Los autores)

\begin{tabular}{|c|c|c|c|c|}
\hline \multicolumn{1}{|c|}{$\begin{array}{c}\text { Altura } \\
\mathbf{m s n m} \\
\mathbf{( m )}\end{array}$} & $\begin{array}{c}\text { Temp Min } \\
\text { Promedio } \\
\left({ }^{\circ} \mathbf{C}\right)\end{array}$ & $\begin{array}{c}\text { Temp. } \\
\text { Promedio } \\
\left({ }^{\circ} \mathbf{C}\right)\end{array}$ & $\begin{array}{c}\text { Temp Max } \\
\text { Promedio } \\
\left({ }^{\circ} \mathbf{C}\right)\end{array}$ \\
\hline Quito (Ecuador) & 2850 & 7.1 & 13.8 & 21.1 \\
\hline Cajamarca (Perú) & 2750 & 2.6 & 13.0 & 21.2 \\
\hline Bogota (Colombia) & 2640 & 7.2 & 13.6 & 19.4 \\
\hline Cuenca (Ecuador) & 2550 & 8.0 & 14.4 & 21.8 \\
\hline Pasto (Colombia) & 2527 & 9.9 & 15.2 & 20.4 \\
\hline Loja (Ecuador) & 2060 & 11.3 & 16.5 & 22.8 \\
\hline
\end{tabular}

Bajo esta motivación, y considerando que se han desarrollado varios estudios previos en esta ciudad, se reúnen los datos detectados en distintos análisis de investigadores locales, de los que se consideran los más significativos. A partir de ello se desarrolla un análisis conjunto para concluir las mejores posibilidades para lograr un máximo autoabastecimiento energético urbano. Se presenta una descripción de diferentes tecnologías con capacidad de convertir la energía proveniente de recursos existentes. Se 
describen los principales indicadores que permiten determinar el potencial del recurso y luego se analiza de forma conjunta con la tecnología de conversión existente para tener una evaluación global, determinándose con ello el potencial de autogeneración frente a consumos urbanos. De la solución y predimensionamiento inicial se analiza concretamente la fuente solar en integración en distintas edificaciones, discutiendo implicancias arquitectónicas, de desbalance de producción-consumo y ello frente a la red, considerando la conexión de microgeneradores. Ello permite concluir hacia donde se debería dirigir la planificación energética urbana para lograr un máximo auto aprovisionamiento y además, cómo los resultados hasta ahora determinados permiten establecer posibilidades y estándares energéticos alcanzables en edificaciones considerándolas como fuentes de energía, como alternativas a investigar de forma detallada a futuro.

\section{METODOLOGÍAS APLICADAS}

\section{En el contexto del metabolismo urbano}

En esta sección se describen las metodologías que se han aplicado desde instancias iniciales durante el proceso investigativo, el cual arrancó en el año 2016. Para establecer de manera aproximada un potencial energético desde fuentes renovables de forma conjunta. La primera instancia es descifrar en campo a través de técnica multicriterio PROMETHEE [21] conjuntamente con revisión bibliográfica, las tecnologías renovables aplicadas a ámbitos urbanos, utilizando recursos disponibles dentro de sus límites. Se descifra con ello que cinco poseerían mayor oportunidad en la ciudad estudiada, más una que ya está implementada; el detalle de este proceso puede revisarse en estudios previos desarrollados por Barragán-Escandón y otros [4,22]. Al ser determinados aspectos como aceptación e integrabilidad social se detecta además del potencial bruto condiciones que se han desarrollado ampliamente en otros contextos [23,24] aspectos detallados del potencial técnico [25]. Para definir el impacto de estas tecnologías se empleó el modelo LEAP (Long Range Energy Alternatives Planning) [26], con esta herramienta y análisis de modificaciones por la incursión de una o varias fuentes se halla un balance entre producción y consumo. Los resultados se encuentran publicados a detalle en los estudios de Barragán y otros [27][28].

Ya en análisis individuales, para determinar el potencial de obtención de energía térmica a baja temperatura, desde tecnología Solar Térmica (ST) se aplican indicadores de consumo frente a ocupación espacial requerida para implementación de esta tecnología. Se descifra que la superficie urbana requerida de captación solar (instalación de placas térmicas) no resulta en una restricción significativa y no es un limitante, por cada persona se requiere una superficie de $0,5 \mathrm{~m}^{2}$ lo que cubriría mayormente el consumo individual considerando consumos típicos residenciales. Ello implica que solamente en edificios en altura no alcanzaría a existir la superficie mínima requerida para cubrir la demanda con estas instalaciones debido a la baja relación de techo por habitante. Por otra parte, en necesario establecer que no es práctico el plantear sistemas ST para abastecer la totalidad de abastecimiento térmico sin sistema de respaldo, porque ello supondría instalaciones sobredimensionadas, con utilidad solo en el peor de los escenarios (baja irradiación y el extremo máximo consumo). La energía de respaldo no obstante pude ser electricidad 
reduciendo así la necesidad de combustible en las edificaciones para calentar agua, por lo general Gas Licuado de Petróleo (GLP). Así, considerando la eficiencia de colectores y población a abastecer, se determina un indicador de contribución solar. La metodología a detalle está desarrollada por E. Barragán-Escandón, [29].

En tercera instancia, se estima el potencial de generación de pequeñas hidroeléctricas potencialmente instalables sin represar los ríos urbanos. Para ello se aplica una metodología que permite calcular la generación eléctrica a partir de caudales, información existente para la ciudad, aplicando a partir de ello indicadores de energía potencial existente por el desnivel existente en el transcurso de los cauces. La metodología a detalle está desarrollada por E. Barragán-Escandón[29].

Otra alternativa adoptada es la generación eléctrica a partir de residuos sólidos urbanos. Es necesario establecer que es la única tecnología en funcionamiento actualmente. Para considerar esta capacidad se utiliza los rendimientos actuales de la planta de procesamiento de Pichacay, que alcanza a producir sobre los 7 GWh/año [30]. Es necesario considerar en este caso la generación si bien está fuera de los límites urbanos, la fuente energética si está dentro de los límites urbanos.

La cuarta opción analizada es generación desde recursos de desechos forestales para generación eléctrica. Para ello a partir de toma de datos y determinación de capacidad energética de residuos forestales leñosos, se mide la carga energética de la vegetación actual. A partir de ello y de toma de datos de podas municipales urbanas, se determina la carga energética útil, extrapolando los resultados de zonas puntuales de muestra de las que se obtiene efectivamente la carga energética forestal, se proyecta a la totalidad de superficie disponible a partir de información municipal de zonas verdes en Sistema de Información Geográfica (GIS). La metodología y los datos de cantidad existente del recurso se encuentra descrito y detallado en la investigación desarrollada por Urgiles \& Yanez, [31],

La siguiente alternativa que se analizó es la generación eléctrica a partir de biogás obtenido de lagunas de oxidación de aguas residuales. A partir de eficiencia de producción y recolección del biogás formado en el proceso de depuración de este equipamiento purificador ya existente en la ciudad de Cuenca. A partir de eficiencias determinadas en estudios internacionales y el volumen estimado disponible de aguas residuales, se dimensiona una posible generación energética para aprovisionar transporte público. El detalle de la metodología está descrito por E. Barragán-Escandón, [29].

En siguiente instancia de este trabajo se entrega una posible autogeneración desde distintas fuentes: Para el potencial fotovoltaico (PV) que se ha detectado como la tecnología de mayor aceptación inicial, se aplica una estimación de superficies de techumbre desde información municipal e indicadores determinados en estudios referenciales. Considerando que en Cuenca, existe una gran preponderancia de techumbres inclinadas y que particularmente en esta latitud, el potencial de generación eléctrica es elevado en cualquier superficie inclinada, sin importar la orientación, se considera que todos los faldones son útiles para aplicación de PV. Por ello se realiza en estudio previo la determinación de rendimiento PV en Cuenca, para así poder calibrar un modelo desde irradiación y archivo climático cuya fuente es la Universidad Politécnica Salesiana y calibración para la herramienta desarrollada cuyo proceso y validación en 
concordancia con rendimiento PV está descrita a detalle por Izquierdo-Torres, Pachecoportilla, Gonzalez-Morales, \& Zalamea-Leon [32]. Desde este rendimiento y aplicando índices de ocupación, sombras, pérdidas de conexión aplicadas a los rendimientos PV, se establece un potencial rendimiento anual.

\section{El potencial solar urbano}

Una vez determinado las potencialidades de cada alternativa con los resultados que se mostrarán en la siguiente sección, se busca determinar en posibilidades reales y de forma más detallada basado en rendimiento. Se estableció la fuente solar PV como una de las opciones con mayor capacidad como se describirá en la sección subsiguiente. Por ello se describe el potencial en distintas tipologías de edificaciones, áreas urbanas e incidencia arquitectónica. Se levantan techos de edificaciones residenciales, industriales, multifamiliares o de servicio y se compara con consumos reales. Para esto ha sido fundamental la información entregada por el distribuidor de electricidad local a los autores [33]. En el mismo orden se deja planteado la exploración de rendimiento de colectores ST, buscando el determinar la validez del modelo F-Chart [34] en su rendimiento efectivo, bajo condiciones ambientales particulares ecuatoriales, con un recorrido solar particular.

\section{RESULTADOS}

\section{Potencial energético con recursos endógenos}

Desde información proporcionada por la distribuidora eléctrica local más la información del centro de distribución de hidrocarburos, se ha podido establecer para el año 2015, los destinos energéticos urbanos (Figura 1). La imagen muestra la distribución de consumos convertidos a Miles de Barriles Equivalentes de Petróleo (KBEP), así como las fuentes de procedencia primaria y secundaria. Los resultados marcan particularidades, por ejemplo, la significativa preponderancia del transporte y reducido consumo residencial como aspectos resaltantes considerando condiciones habituales publicados en reportes de la IEA [35] para otros países, que señalan que el transporte alcanza por lo general un $40 \%$ de consumo, y las edificaciones el $41 \%$. Sin embargo, el transporte en Cuenca es casi del $60 \%$ y en edificaciones el 34\%. Esta diferencia, con respecto a otros contextos, se puede explicar por el mínimo consumo comparativo de las edificaciones para abastecer demandas de acondicionamiento ambiental, frente a edificaciones en países estacionales en los cuales los requerimientos energéticos para ambientación, gracias al clima favorable, condición explicada previamente. Contrariamente, en cuanto a transporte, el alto consumo es consecuencia un alto índice de transporte particular a combustión, situación impulsada por el subsidio a combustibles, por ello se espera que este se mantenga como la demanda más alta de combustibles en los próximos años [36]. 


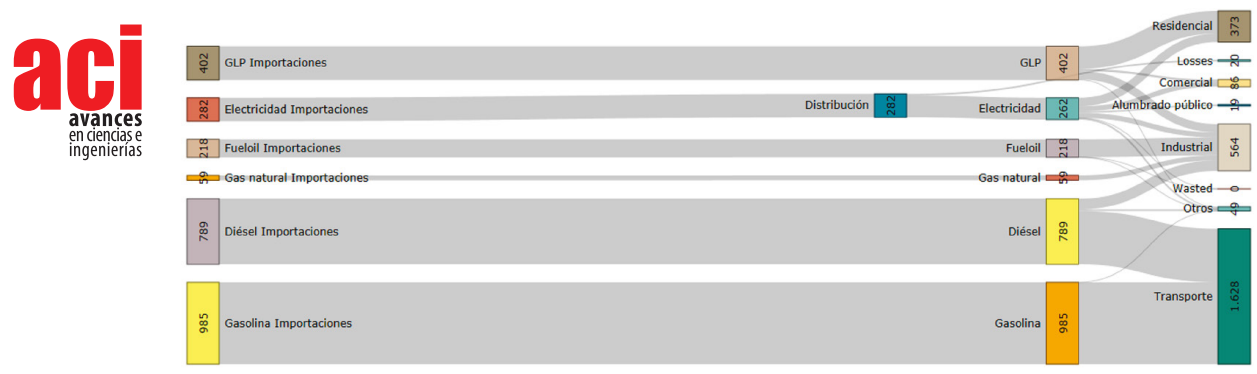

Figura 1: Diagrama de Sankey de consumos energéticos urbanos de Cuenca (en KBEPS) (Los autores [33])

Una vez desarrollados los estudios de potencial energético general que se encuentran descritos detalladamente en los documentos E. Barragán-Escandón, Zalamea-León, Terrados, Vanegas-Peralta, et al., [4] y Urgiles \& Yanez, [31], se puede apreciar que el consumo total registrado en el año 2015 es del equivalente 2739 KBEP en electricidad o combustibles fósiles. La electricidad significa apenas el 9,8\% de consumo energético urbano, es una fuente que idealmente debería crecer ya que reduce la necesidad de combustión en sitio y puede ser cubierta desde tecnologías con cero emanaciones. La mayor proporción de demandas está cubierto por combustibles y combustión desarrollada dentro de los límites urbanos; de ello la mayor contaminación registrada es por contaminación vehicular, de la cual procede el $85 \%$ de los gases y partículas contaminantes en la ciudad, con impactos no dimensionados a la salud pública [37].

Luego de determinar los consumos, estos sirven de base para establecer el potencial energético desde las fuentes energéticas urbanas. Para ello se plantea un escenario de destinar el potencial PV, mini-hidro, residuos sólidos urbanos y poda de residuos forestales para aprovisionamiento eléctrico. La tecnología ST para abastecer agua caliente sanitaria (ACS); biogás procedente desde aguas residuales destinado a abastecer el consumo de transporte público. La Figura 2 muestra el resultado de la generación alternativa con recursos endógenos, distribución en potencial anual, de la cual se puede encontrar más detalle en la tesis E. Barragán-Escandón, [29]. Marcadamente el recurso de mayor potencial es el sol, y concretamente la tecnología PV, la cual conjuntamente con las otras fuentes alcanzaría a abastecer la totalidad de consumo eléctrico urbano, que corresponde al 9,8\% del total. No obstante, existe un excedente de 736 KBEP, los cuales serían remanentes eléctricos no utilizables bajo situación actual. Entonces, el potencial solar total es 3 veces superior a las mediciones de consumo eléctrico, ello significa que se puede alcanzar un mayor margen si el consumo de combustibles se transfiere tecnológicamente a alternativas de alimentación eléctrica. La superficie disponible neta para introducción PV en techumbres ha sido establecida acorde a información de predios y ocupación espacial de edificaciones a la que se aplican indicadores de generación, irradiación disponible, afectaciones arquitectónicas, etc.; detallado por Antonio Barragán-Escandón, Zalamea-León, \& Terrados-Cepeda, [27]. Las posibilidades ciertas serían el integrar decididamente la inclusión de cocción con inducción, situación ya analizada en estudios previos [33], así como la inclusión consistente de transporte eléctrico [38]. La tecnología ST puede contribuir además con un consumo del 2,2\% de los consumos térmicos totales urbanos del 2015; si bien no parecería una contribución significativa, es relevante en la reducción del gasto de GLP destinado a calentamiento 
de ACS; es estrategia prometedora para reducir el gasto en subsidios a GLP, política que implica ingente gasto estatal en importaciones además se ser altamente ineficiente [38]. Entre las tecnologías de mini-hidro, generación de desechos sólidos y biomasa apenas supera un $1 \%$ del consumo total urbano, el recurso de podas de hecho resulta insignificante (0,01\% respecto al consumo base) [31]. En la Figura 2 y Tabla 2 se pueden advertir los potenciales de las distintas tecnologías y ello comparado con las demandas de las distintas fuentes.

Analizados estos potenciales globales conjuntos y definidos los de mejor aptitud, se vislumbra que la fuente solar es la más prometedora, tanto PV como ST. A partir de ello, se desarrollan análisis iniciales de integración solar en edificaciones en el ámbito urbano.

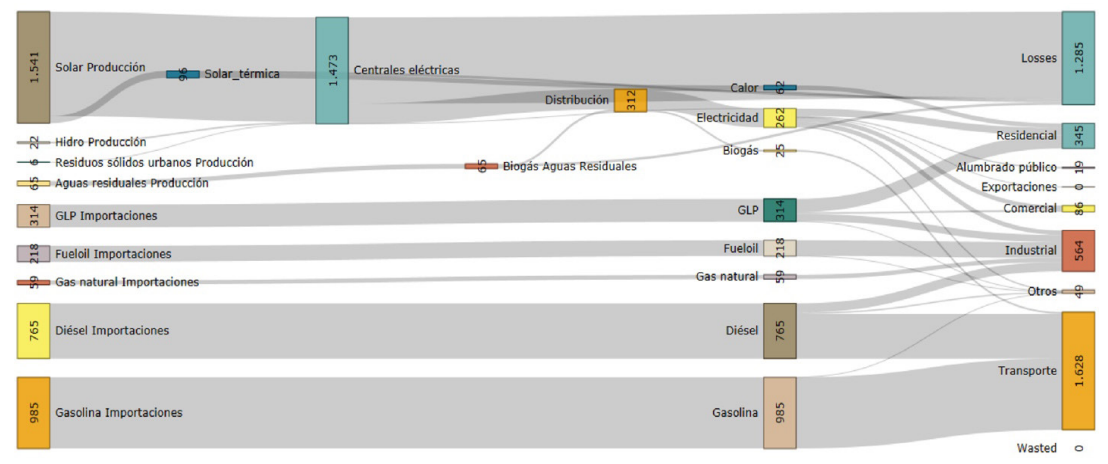

Figura 2: Diagrama de Sankey de consumos con posible auto-aprovisionamiento desde fuentes diversas desde recursos endógenos en Cuenca (KBEPs) (Los autores[32]) 
Tabla 2. Balance energético $\mathrm{E}_{56}(\mathrm{kBEP})$

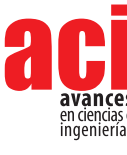

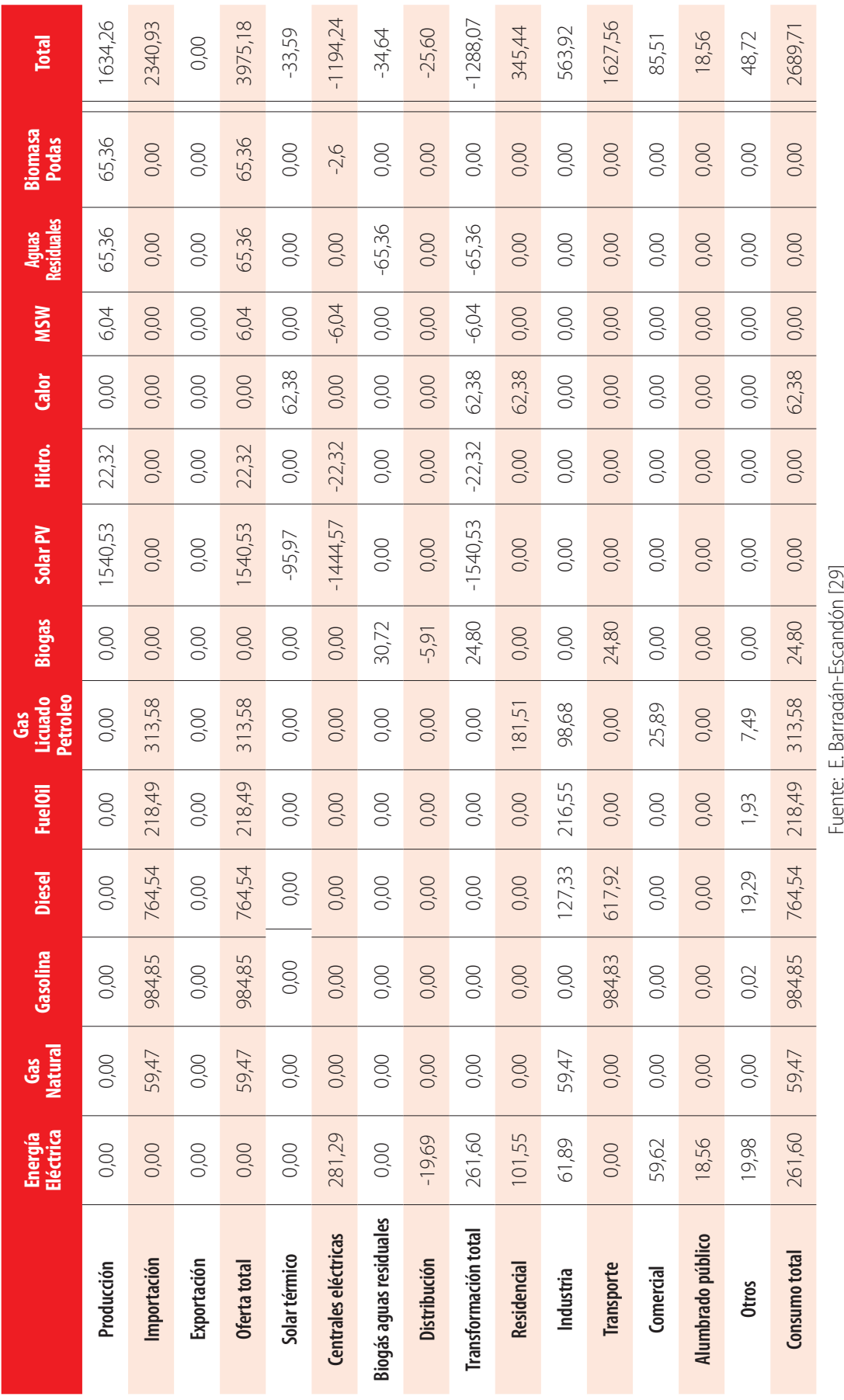




\section{Rendimiento PV en condiciones de integración en techos}

Para establecer la producción PV se descifra el rendimiento de la tecnología. Como se mencionó, en el contexto de estudio existe una marcada preponderancia de techumbres inclinadas, condición característica de locaciones con alta pluviosidad. En condiciones de países localizados en regiones estacionales, se consideran en el hemisferio norte, las fachadas y techos orientados hacia el hemisferio sur presenta irradiación relevante; contrariamente en el sur, aquellas orientadas al norte. Por ello el primer análisis es encontrar qué implican las condiciones de orientación e inclinación de PV en localizaciones tan próximas a la línea ecuatorial. Para ello se analizó previamente el rendimiento de placas PV en distintas disposiciones respecto al firmamento, al tiempo que se recolecta información climática, entonces, a partir de ello con lecturas de rendimiento de períodos cercano de una semana en cada disposición, se calibra modelo de rendimiento PV en herramienta de simulación SAM, (Simulation Advisor Model). El modelo al analizar frente a producciones reales muestra buena fiabilidad bajo distintas condiciones de irradiación, acorde a resultados comparativos desarrollados por investigadores locales [32].

Los resultados significativos son entre otros la existencia de un elevado rendimiento PV en cualquier orientación, sobre todo cuando la inclinación de los PV es baja. Con el modelo de SAM establecido y archivo climático se detecta que la mayor producción se obtiene con orientación SE y la mínima al NO, no obstante, en balance anual es apenas 1,5\% la primera sobre la segunda, en estudio en inclinaciones entre $0{ }^{\circ}$ y $30^{\circ}$ de pendiente, en donde también se determina que la proporción de esta tipología de techos supera el 90\% [33]. Se concluye entonces que cualquier techumbre inclinada en estas latitudes tiene potencial sustancial si esta no está afectada por sombras, limitación que también es menor en esta latitud respecto a otras por configuración urbana y recorrido solar.

\section{Potencial solar en tipologías urbanas}

\section{Potencial PV en edificaciones bajas mixtas}

Un primer análisis se hizo considerando una muestra representativa de una de las tipologías de casas. Se adoptaron viviendas/edificaciones típicas del centro histórico comprendidos en 80 manzanas del núcleo central de la ciudad y entre estas, 119 edificaciones individuales. Por tratarse del centro de la ciudad, estas presentan uso mixto principalmente residencial, comercial y de oficinas. Este trabajo fue auspiciado por la empresa de distribución eléctrica la que entregó curvas de consumo de los sectores en donde se localizan estas viviendas. El consumo medido neto anual del conjunto de casos estudiados está en el orden de 495 MWh. Frente a ello la producción neta con PV monocristalino que se adaptarían arquitectónicamente, se estima en 2815 MWh / anual, es decir, 5,7 veces el consumo base. Además, por condiciones arquitectónicas se estima el rendimiento con teja PV que sería de $1059 \mathrm{MWh} / a n ̃ o$, esto corresponde a 2,1 veces a la energía utilizada. Este potencial refleja que superficies de techumbre en esta tipología, exceden la demanda. Con los excedentes, por ejemplo, sería posible el energizar autos eléctricos para un recorrido de $387.000 \mathrm{~km}$ con la instalación masiva de placas monocristalinas, considerando un rendimiento de los vehículos de $6 \mathrm{kWh} /$ km [39] . La condición de ecuatorialidad y de mínimos consumos urbanos registrados suponen este potencial. Los resultados reflejan además indicadores de una producción 
posible de $202 \mathrm{kWh} / a n ̃ o ~ p o r ~ \mathrm{~m}^{2}$ en placa PV de sílice monocristalino, $59 \mathrm{kWh} / \mathrm{año}$ por $\mathrm{m}^{2}$ de teja PV. Ello entrega indicadores de producción asociados de generación por m² de techumbre neto de 143,30 kWh al año, lo que implica una eficiencia neta de 9,3\% en el caso de placas de sílice y 3,5\% de eficiencia al aplicarse tejas PV. Es Ilamativo, además, que una familia integrada por tres a cuatro personas, el consumo está en el orden de $140 \mathrm{kWh} / \mathrm{mes}$ a $160 \mathrm{kWh} /$ mes, observando el rendimiento de un $\mathrm{m}^{2}$ sugiere que en estos casos, para alcanzar un equilibrio respecto al consumo anual implica alrededor de 10 a $12 \mathrm{~m}^{2}$ de placas PV cristalinas de 60 celdas. Además, se pudo advertir que es necesario considerar los desbalances momentáneos de consumo-generación, el análisis se pudo realizar en margen horario en los días de máxima, mínima e irradiación promedio; no obstante, las oscilaciones para diseño de redes deben considerarse con mayor resolución ya que las fluctuaciones pueden darse en minutos, incluso segundos con caídas e incrementos tanto de producción como consumo. (Figura 3).

En este estudio se realizaron diversos análisis de producción respecto a consumos contabilizados. Uno de estos casos es considerar, en el promedio de los 119 casos, una dimensión de instalación PV considerando una cantidad requerida para alcanzar el estándar Net-Zero desde el consumo eléctrico. Este estándar (una de sus definiciones) supone que una edificación alcanza a generar cuanta energía consume en balance anual [40]. Suponiendo este escenario, en la Figura 3 se refleja, con un índice de ocupación de 17,5\% de superficie de techo en promedio se alcanzaría teóricamente dicho estándar; si analizamos la producción con dicha superficie en días de máxima, mínima e irradiación promedio (se tomaron días registrados y su irradiación en el 2016), se observa que para alcanzar este estándar, existiría excedentes (energía en exceso) a entregar a la red en las horas cercanas al medio día; solamente días de baja irradiación no alcanzaría o existirían mínimos excedentes. Ello supone la necesidad de explorar incidencia en la red de integración de PV a escala en el ámbito urbano. Este y otros escenarios que pueden resultar de interés, así como la metodología aplicada, es descrito a detalle por Zalamea y otros [33].

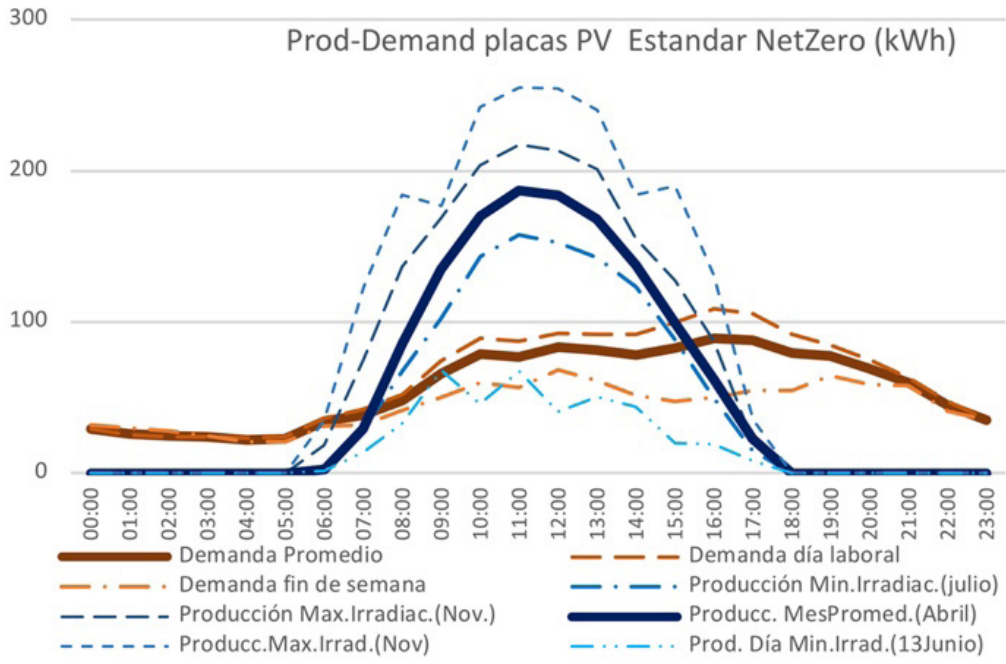

Figura 3: Curvas horarias de producción consumo considerando indicador de ocupación PV Net-Zero (Los autores[46]) 


\section{Potencial PV en equipamiento industrial}

Una zona especialmente sensible a la contaminación y al consumo energético es el denominado "Parque industrial" de la ciudad de Cuenca. Para analizar el desempeño y potencial autoabastecimiento de esta tipología con condiciones de particular potencial autoaprovisionamiento, se toma la industria con mayor consumo y con mayor ocupación en extensión del conjunto urbano. A su vez esta está compuesto por edificaciones individuales con extensos techos con inclinación baja, regulares geométricamente, es decir, con características idóneas para integrar PV. El complejo está en un predio de casi 10 Hect. de terreno y ocupa poco más de 5 Hect con edificaciones, en su gran mayoría de producción y almacenamiento de producto. Para este estudio se realiza un diagramado tridimensional del complejo y fundamentalmente de su techumbre. Se analiza las condiciones arquitectónicas locales, con énfasis en cuanto a requerimientos de iluminación cenital y ventilación como prioridades arquitectónicas. Luego de establecerse estas condiciones, se plantea una ocupación máxima de PV en la techumbre de esta industria. Se alcanza con ello a integrar 28516 placas de sílice cristalino de 60 celdas lo que significaría 41633,36 m² de superficie de captación solar (Fig. 4).

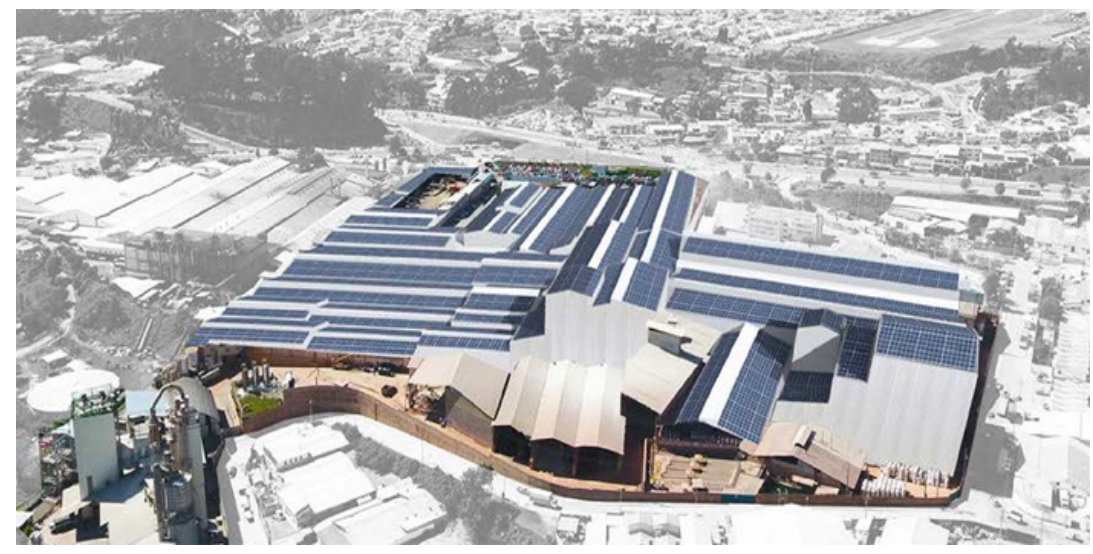

Figura 4: Fotomontaje de propuesta de integración de $41600 \mathrm{~m}^{2}$ de captación PV en edificio industrial en Cuenca, Ecuador (los autores [48])

A partir de la superficie disponible, con el modelo SAM establecido previamente se obtiene el rendimiento eléctrico factible acorde a las distintas inclinaciones y orientaciones de los faldones. Con datos de los consumos eléctricos horarios provistos por la empresa de distribución del año 2016 y con archivo de condiciones climáticas de ese mismo año, se simula la producción factible frente a los consumos. El análisis horario se muestra en la Fig. 5. Se encuentra que a pesar d existir una capacidad dimensional para instalarse más de 4 Hect. de PV, apenas se alcanza a cubrir el 22\% del consumo actual. A pesar de observarse un porcentaje de abastecimiento que no alcanza la cuarta parte, en momentos de alta irradiación que coinciden con bajos consumos, se pueden esperar excedentes de potencia de sobre los $3 \mathrm{MW}$ por momentos. Ello supone que también existe requerimiento de conexión a red para aprovechar el potencial completo [41]. 


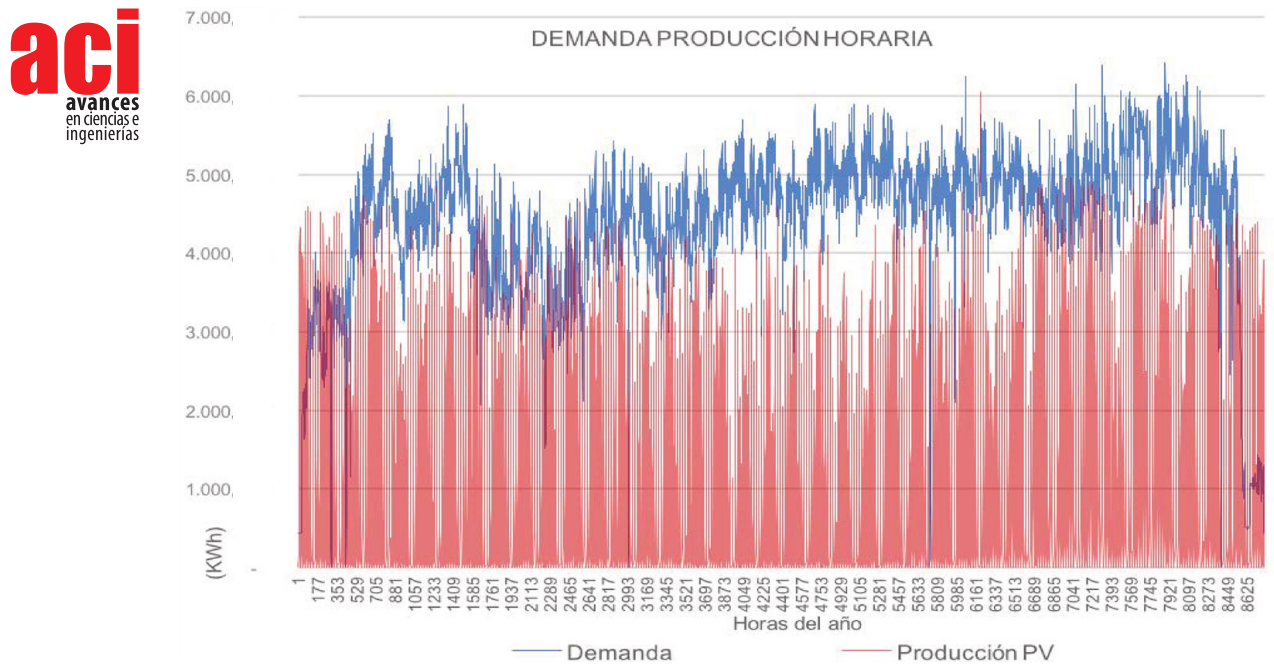

Figura 5: Comparación producción- consumo horario industria de alta demanda de Cuenca (los autores[41])

\section{Incidencia en red}

Por lo anterior, se ve la necesidad de profundizar en implicaciones en la red eléctrica. En consecuencia, se ha simulado la capacidad de inclusión PV a gran escala en los techos de un sector urbano alimentado por un transformador de media a baja tensión. Además, se considera la integración en todas las residencias existentes en aquellas edificaciones, la inclusión de sistemas de cocción eléctrica y además sistemas individuales de almacenamiento con baterías de litio residenciales. El proceso realiza simulaciones de producción y consumo de 119 edificaciones o puntos de carga, así como 119 unidades de potencial generación PV en los techos. A diferencia del potencial solar descrito previamente, este se realiza con una resolución de 15 min, justamente porque se requiere analizar fluctuaciones temporales a mayor escala. Le descripción metodológica detallada, herramientas y resultados están publicados en el trabajo de Zambrano-Asanza, ZalameaLeón, Barragán-Escandón, \& Parra-González [42]. Se concluye que la producción PV tiene el potencial bruto para cubrir la totalidad del consumo de la zona de estudio. Pero al revisar detalladamente restricciones en red y coincidencia de producción-consumo perfecto a nivel del punto de carga, se observa que no es posible abastecer la totalidad del consumo eléctrico medido incluso integrando almacenamiento a través de baterías de litio, pero la proporción alcanzable es significativa de todos modos. Los resultados para esta zona analizada son parciales, pero entregan información importante.

Se simuló autogeneración por puntos de carga individuales (edificaciones), considerando se trata de red eléctrica existente, bajo sus condiciones. La posibilidad de inyectar el exceso de generación en determinado punto de carga adiciona complejidad a la operación y preparación de la red. Predecir la carga y producción de esta fuente fluctuante, es un desafío para la planificación eléctrica, existe la necesidad de instalar sensores de variables externas referentes a niveles de radiación, viento y clima; además contar con herramientas de predicción, simulación y optimización, integradas a la gestión y operación. No obstante, por las condiciones ambientales y de consumo favorables y estables que se dan en Cuenca, muy particulares en el mundo, la energía solar presenta un potencial significativo [43]. 
La evaluación presentada determina que hay oportunidades reales de autoabastecimiento eléctrico con producción cercana a la carga. Si bien no se alcanza a lograr el abastecimiento teóricamente alcanzable en escenarios con conexión en redes inteligentes y almacenamiento (de hasta un 90\%), el porcentaje estimado es muy relevante de hasta un $46 \%$ sin almacenamiento y hasta $73 \%$ con almacenamiento considerando incluso consumos incrementados pero coherentes con la producción solar como la cocción. Estos resultados son superiores a otras situaciones latinoamericanos que adolecen por ahora de la capacidad técnica en red, en los que se prevé una fracción solar de máximo hasta 27\% sin almacenamiento o 35\% con almacenamiento [6] como consecuencia de clima y oscilación de irradiación estacional.

Son además deseables escenarios alternativos para maximizar un autoaprovisionamiento, es la posible compra-venta a red y almacenamiento a gran escala en media tensión y exportación en red a otras zonas urbana (comerciales, servicios e industriales) bajo condiciones espacio-temporales en Smart Grid [44]. También la adopción de estrategias para maximizar coincidencia producción-consumos típicos, programando las demandas estratégicamente a nivel de consumidor [44]. La Fig. 6 muestra la simulación de producción (superior) consumo (imagen inferior) de los 119 casos.
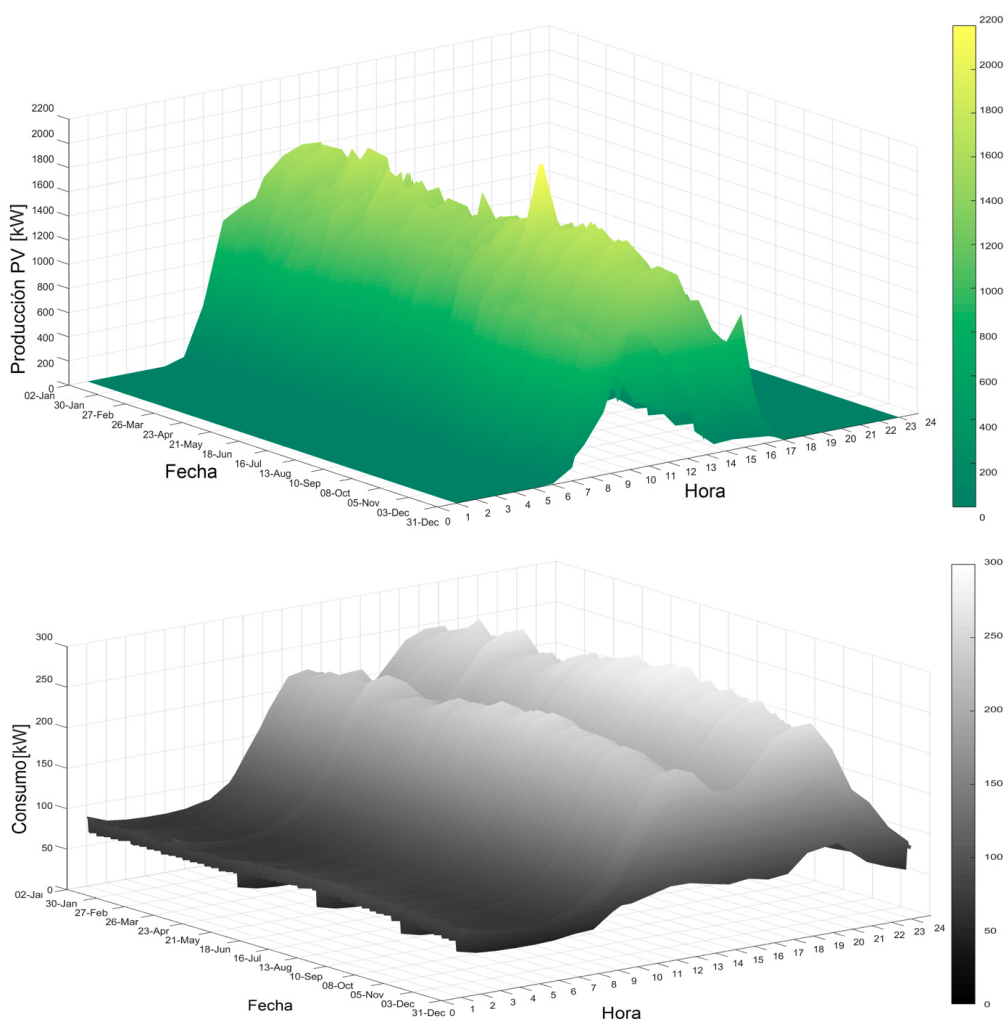

Figura 6: Análisis anual de producción PV frente a consumos de 119 edificaciones con integración PV en curvas horarias-año (Los autores [42]) 


\section{Implicancia arquitectónica}

Al mismo tiempo, se ha venido desarrollando inicialmente un análisis de las consecuencias ambientales de la integración arquitectónica en edificaciones. Si bien este análisis se ha hecho conjuntamente con el rendimiento PV, también se torna aplicable a los colectores ST cuando estos no tienen integrados sistemas de almacenamiento, ya que ello provocaría resultados aspectuales similares. La zona más sensible en este aspecto sería el Centro Histórico de Cuenca, zona Patrimonio Cultural de la Humanidad con edificaciones valoradas. Cabe recalcar que en el estado del arte, en edificaciones en centros urbanos de ciudades y zonas con mayor valor en el mundo (medievales), como son ciudades europeas, incluso en el Vaticano, se ha integrado PV, llevando el aspecto ambiental preponderancia a pesar de los valores estéticos-patrimoniales. Se trata justamente de uno de los aspectos con más amplia discusión actual [45].

Para llevar este análisis en el contexto de Cuenca, se desarrolla la investigación no solo desde el punto del potencial solar, sino se relaciona las implicancias arquitectónicas resultantes. A partir de la comparación de estos dos aspectos se ha definido que las superficies de techo exceden la necesidad espacial para alcanzar el estándar Net-Zero. De hecho, dependiendo del caso de estudio en el análisis desarrollado se encuentra que con una ocupación de entre el 20\% y 5\% de ocupación en techo con placas de sílice se puede abastecer los consumos energéticos de estas edificaciones; el análisis completo se desarrolla en E. Zalamea y otros. Así también por condiciones de red se podría ocupar espacialmente entre el 16\% de superficie disponible para suplir los consumos actuales; $y$ hasta un 30\% considerando la instalación masiva de cocinas eléctricas y almacenamiento con baterías de litio [39]. Se concluye en estos trabajos que las superficies de captación pueden ubicarse en sectores de techumbre que no impactan de manera directa al ambiente urbano. De hecho, considerando la escala de calles, los PV serían perceptibles desde visuales cercanas, únicamente en el caso de edificaciones de una planta en contacto directo con el espacio público. De ahí en más los dispositivos solares serían perceptibles desde visuales lejanas y sobre todo aéreas; más allá de ello, en todo el centro histórico se observan otros tipos de techos que no son de teja que superan la superficie requerida para alcanzar el estándar Net-Zero, o además de otros tipos de elementos igual o más impactantes como tragaluces o antenas. En las Figuras a continuación, se pueden observar dos edificaciones con la cantidad de PV requeridos para alcanzar el estándar NetZero de aquellos casos puntuales, con mediciones reales de los dos casos; en el caso con mayor consumo (Fig. 7) en donde funciona un museo durante todo el día se requiere 56 placas de sílice; en el segundo (Fig. 8) una vivienda y comercio menor requiere solamente 11 placas. En ambos casos mostrados en fotomontaje, las placas PV de sílice serían observables solamente desde vistas aéreas, de estar dispuestas en los faldones posteriores no orientados al espacio público. 


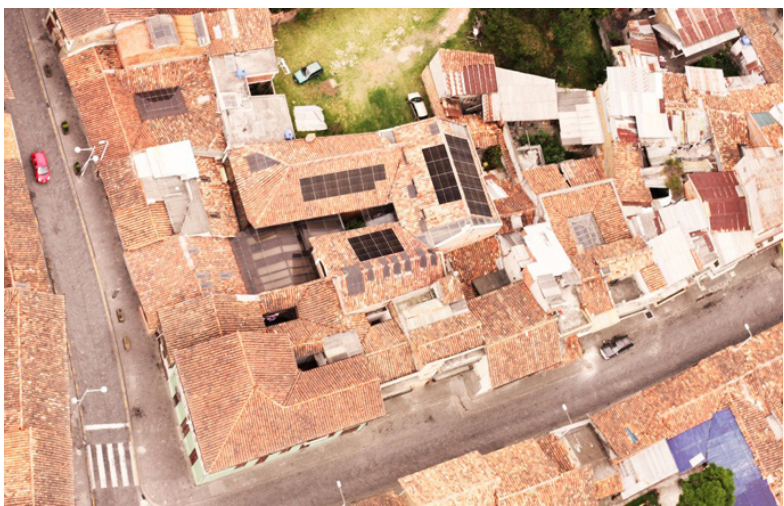

Figura 7: Edificaciones con montaje de placas requeridas para alcanzar el estándar Net-Zero de alto consumo (Esteban Zalamea-León / Antonio Barragán-Escandón [39])

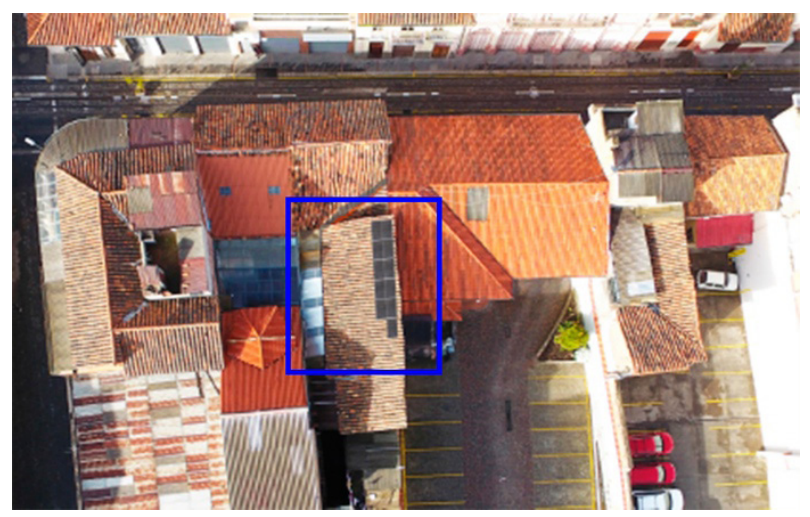

Figura 8: Edificaciones con montaje de placas requeridas para alcanzar el estándar Net-Zero de consumo promedio (Esteban Zalamea-León / Antonio Barragán-Escandón)

\section{CONCLUSIONES Y RECOMENDACIONES}

Este artículo se trata de una revisión de varios trabajos realizados en investigaciones desde 2015 desarrolladas y publicadas, describiendo posibilidades y magnitudes para lograr un eventual abastecimiento con recursos endógenos y renovables. Las ciudades como fuente de su propia energía son una situación ideal desde la perspectiva ambiental. La coyuntura actual que muestra la necesidad urgente de reducir y eliminar subsidios a los combustibles fósiles exige el promover alternativas que además posibiliten generar puestos de trabajo en lugar de gastar ingentes recursos en importaciones energéticas que además resultan ineficientes, contaminantes y con usufructo de corporaciones extranjeras. Se plantea la situación bajo el escenario de consumos de años resientes. La limitación de este trabajo en su verdadera implementación es sin duda que la base 
de este estudio se vería modificado para los años subsiguientes, por lo que lograr lo planteado de forma precisa resultaría en otras magnitudes. La intención es mostrar el potencial actual, para promover las mejores posibilidades a futuro.

La primera parte recopila los resultados obtenidos a partir de seis fuentes energéticas en los que se denota como aspecto primordial que, si bien entre todas las posibilidades existe el potencial de abastecer algo menos de un 14\% del consumo base, existirían excedentes para abastecer una magnitud de tres veces los requerimientos eléctricos y podría alcanzar sobre el 38,8\%, el potencial eléctrico total es 998 KBEP, pero el consumo eléctrico urbano solo 262 KBEP. Pero para ello, se debe tornar útil este excedente, siendo necesaria la conversión de consumos actuales alimentados por combustible fósiles a eléctricos. Los estudios muestran que estratégicamente se deben focalizar en la sustitución de los energéticos destinados al transporte, que actualmente es el sector con mayor consumo y más contaminante; aunque además la cocción o abastecimiento de ACS también puede ser significativo.

En segunda instancia, a partir del excelente potencial determinado (las dos fuentes con mayor capacidad son la PV en primer lugar y la ST en segundo) se desarrolla investigación puntual de la primera opción. En cuanto a despliegue por disposición PV es destacable el relevante potencial existente en cuanto a captación, cuando los PV presentan pendientes concordantes con las techumbres típicas locales, existiendo excelente irradiación con mínima afectación por condiciones de orientación; condición particularmente positiva de esta latitud. En el caso de viviendas se observa que se requiere, dependiendo el caso, de márgenes menores para alcanzar el estándar Net-Zero; normalmente en un rango de entre el 5\% al 20\%; en el caso de la industria, si bien por el elevado consumo. Se obtiene una fracción, no obstante, es una importante producción, concretamente en la industria analizada se podría instalar más de 4 hect. de PV, lo que representa el consumo eléctrico promedio de 17000 cuencanos. Es necesario el seguir con investigaciones en otras tipologías y barrios urbanos particulares, no obstante, los análisis expuestos permiten al lector tener una idea, de la capacidad PV urbana, en el contexto particular. También por las buenas condiciones climáticas con mínima variación estacional, se observa excelentes condiciones para integración a red, incluso en condición actual, sin requerir tecnología de redes inteligentes. Sin embargo, para poder exportar excedentes entre zonas de la ciudad, se debe reacondicionar la red de forma que maneje flujos espacio temporales entre zonas de elevada producción (áreas residenciales), a zonas de alto consumo, como industriales, de equipamiento o comerciales.

La segunda tecnología solar, la ST está en análisis como trabajo futuro. Si bien ha se ha predeterminado existiría un potencial de hasta un 2,2\% del consumo urbano, en el caso de edificaciones residenciales, este abastecimiento se espera alcance márgenes superiores al requerimiento de energía para abastecer ACS. Es una alternativa ideal como complemento a la cocción por inducción justamente como estrategias para reducir los actuales subsidios y así descarbonizar la matriz energética actual. Concretamente se analizará el rendimiento de los colectores ST de tubo de vacío (heat pipe) y de placa plana, para validar modelos de simulación, así como establecer la tecnología con mejor aptitud.

Los análisis futuros están encaminados a determinar posibilidades y requerimientos para alcanzar los estándares Net-Zero (consumo total energético de una edificación 
igual a la energía generada) Plus-Energy ( estándar en el que la edificación genera más de lo que consume para suplir usos urbanos y de transporte) y el más reciente que es el estándar Power-Building nueva definición para edificaciones que generan la cantidad de energía ocupada durante la operación de la edificación más la energía contenida en materiales y proceso de construcción y deconstrucción en un balance a sesenta años. Estos estándares son complejos de alcanzar especialmente en los climas adversos, en donde se requiere cuantiosa carga energética para lograr confort, además de exigencias altas en construcción y aislamientos que además provocan más energía contenida. En el clima ecuatorial andino con estrategias sencillas, control de infiltración y buena captación pasiva más ventilación se puede alcanzar niveles de confort sin necesidad de equipos de ambientación o consumo energético alguno. Sin esta carga, se concluye finalmente, que uno de los lugares del mundo en donde es más fácil alcanzar los estándares descritos es en la situación geográfica de la ciudad analizada y cualquier ciudad bajo condiciones climáticas similares; de estas hipótesis, también surgen evidentemente la necesidad de ampliar la investigación.

\section{AGRADECIMIENTOS}

Este trabajo forma parte del Proyecto de investigación "Calibración de modelo F-Chart para colectores solares térmicos con parametrización y validación acorde a disposiciones típicas para integración arquitectónica en climas ecuatoriales andinos". Es financiado por la Dirección de Investigación de la Universidad de Cuenca, a la cual enviamos el más profundo agradecimiento.

\section{CONTRIBUCIÓN DE LOS AUTORES}

Contribuciones de E. Zalamea, estructuración y recopilación de información. Es autor principal de las investigaciones revisadas de potencial solar urbano y de edificaciones. Contribuciones de A. Barragan, fue el autor de la metodología y obtención del potencial renovable urbano desde distintas fuentes y revisor del potencial solar en conjunto con el autor principal. 


\section{REFERENCIAS}

[1] Byrne, J., Taminiau, J., Seo, J., Lee, J., \& Shin, S. (2017). Are solar cities feasible? A review of current research. International Journal of Urban Sciences, O(0), 1-18. https://doi.org/10.1080/12265934.2017.1331750

[2] Brown, T. W., Bischof-Niemz, T., Blok, K., Breyer, C., Lund, H., \& Mathiesen, B. V. (2018). Response to'Burden of proof: A comprehensive review of the feasibility of $100 \%$ renewable-electricity systems.'Renewable and Sustainable Energy Reviews, 92(September 2016), 834-847. https://doi.org/10.1016/j.rser.2018.04.113

[3] Mosannenzadeh, F., Bisello, A., Diamantini, C., Stellin, G., \& Vettorato, D. (2017). A case-based learning methodology to predict barriers to implementation of smart and sustainable urban energy projects. Cities, 60, 28-36. https://doi.org/10.1016/j.cities.2016.07.007

[4] Barragán-Escandón, E., Zalamea-León, E., Terrados, J., \& Vanegas-Peralta, P. (2019). Factores que influyen en la selección de energías renovables en la ciudad, 45(134), 259-288

[5] Nejat, P., Jomehzadeh, F., Mahdi, M., \& Gohari, M. (2015). A global review of energy consumption , 020 emissions and policy in the residential sector ( with an overview of the top ten $\mathrm{CO} 2$ emitting countries ). Renewable and Sustainable Energy Reviews, 43, 843-862. https://doi.org/10.1016/j.rser.2014.11.066

[6] Wegertseder, P., Lund, P., Mikkola, J., \& García Alvarado, R. (2016). Combining solar resource mapping and energy system integration methods for realistic valuation of urban solar energy potential. Solar Energy, 135, 325-336. https://doi.org/10.1016/j.solener.2016.05.061

[7] Aguilar, E. (2019, 0ctober 17). Ecuador muestra que los subsidios fósiles se niegan a morir. EL CEO. Retrieved from https://elceo.com/internacional/ecuador-muestra-que-los-subsidios-fosiles-se-niegan-a-morir

[8] Vargas, F. (2019, October 19). Primer balance tras los desmanes en Santiago: 41 estaciones de Metro con destrozos y 308 detenciones

[9] Moser, D., Lovati, M., \& Maturi, L. (2018). Photovoltaic City: Effective Approaches to Integrated Urban Solar Power. Urban Energy Transition (2nd ed.). Elsevier Ltd. https://doi.org/10.1016/B978-0-08-102074-6.00030-9

[10] REN21. (2017). Renewables 2017: global status report. Renewable and Sustainable Energy Reviews (Vol. 72). https://doi.org/10.1016/j.rser.2016.09.082

[11] Renewable Energy Agency, I. (2018). Renewable Power Generation Costs in 2017. International Renewable Energy Agency. https://doi.org/10.1007/SpringerReference_7300

[12] IEA. (2009). Cities, Towns \& Renewable Energy Cities, Towns. Paris: IEA/OECD. Retrieved from http://www.iea.org/ publications/freepublications/publication/Cities2009.pdf

[13] IRENA. (2018). Renewable Energy and Jobs: Annual Review 2018, International Renewable Agency. https://doi. org/10.1158/1078-0432.CCR-16-2586

[14] Poggi, F., Firmino, A., \& Amado, M. (2018). Planning renewable energy in rural areas: impacts on occupation and land use. Energy. https://doi.org/10.1016/j.energy.2018.05.009

[15] Lin, Z., \& Qi, J. (2017). Hydro-dam - A nature-based solution or an ecological problem: The fate of the Tonlé Sap Lake. Environmental Research, 158(May), 24-32. https://doi.org/10.1016/j.envres.2017.05.016

[16] Caldarelli, C. E., \& Gilio, L. (2018). Expansion of the sugarcane industry and its effects on land use in São Paulo: Analysis from 2000 through 2015. Land Use Policy, 76(January), 264-274. https://doi.org/10.1016/j.landusepol.2018.05.008

[17] Benis, K., Turan, l., Reinhart, C., \& Ferrão, P. (2018). Putting rooftops to use - A Cost-Benefit Analysis of food production vs. energy generation under Mediterranean climates. Cities, 78(February), 166-179. https://doi. org/10.1016/j.cities.2018.02.011

[18] Ziebell, A. C., \& Singh, V. K. (2018). Energy indicator in sustainable urban energy metabolism and challenges. Proceedings of the Institution of Civil Engineers - Energy, 171(1), 26-31. https://doi.org/https://doi.org/10.1680/ jener.17.00010

[19] Connolly, D., Lund, H., \& Mathiesen, B. V. (2016). Smart Energy Europe: The technical and economic impact of one potential 100\% renewable energy scenario for the European Union. Renewable and Sustainable Energy Reviews, 60 , 1634-1653. https://doi.org/10.1016/j.rser.2016.02.025 
[20] Dujardin, J., Kahl, A., Kruyt, B., Bartlett, S., \& Lehning, M. (2017). Interplay between photovoltaic, wind energy and storage hydropower in a fully renewable Switzerland. Energy, 135, 513-525. https://doi.org/10.1016/j.energy.2017.06.092

[21] Mareschal, B. (2013). Visual PROMETHEE manual

[22] Barragán-Escandón, A., Terrados-Cepeda, J., \& Zalamea-León, E. (2017). The role of renewable energy in the promotion of circular urban metabolism. Sustainability (Switzerland), 9 (12). https://doi.org/10.3390/su9122341

[23] Grubler, A., Bai, X., Buettner, T., Dhakal, S., Fisk, D. J., \& Ichinose, T. (2013). Urban Energy Systems. In Global Energy Assessment (GEA) Toward a Sustainable Future (pp. 1307-1400). https://doi.org/10.4324/9780203066782

[24] Zhang, X., Shen, L., Chan, S. Y., \& Yee, S. (2012). The diffusion of solar energy use in UK : What are the barriers? Energy Policy, 41, 241-249. https://doi.org/10.1016/j.enpol.2011.10.043

[25] Child, M., Breyer, C., \& Haukkala, T. (2017). The Role of Energy Storage Solutions in a 100\% Renewable Finnish Energy System. Sustainability, 9(1358), 0-25. https://doi.org/doi:10.3390/su9081358

[26] Heaps, C. G. (2016). Long-range Energy Alternatives Planning (LEAP) system. Somerville, MA, USA.: Stockholm Environment Institute. Retrieved from https://www.energycommunity.org

[27] Barragán-Escandón, Antonio, Zalamea-león, E., Terrados-Cepeda, J., \& Parra-González, D. (2019). Las energías renovables a escala urbana. Aspectos determinántes y selección tecnológica. Bitácora Urbano Territorial, 29(2), 39-48. https://doi.org/https://doi.org/10.15446/bitacora.v29n2.65720

[28] Barragán Escandón, A., Terrados Cepeda, J., Zalamea León, E., \& Arias Reyes, P. (2018). Electricity production using renewable resources in urban centres. Proceedings of the Institution of Civil Engineers - Energy, 171(1), 12-25. https://doi.org/10.1680/jener.17.00003

[29] Barragán-Escandón, E. (2018). EL AUTOABASTECIMIENTO ENERGÉTICO EN LOS PAÍSES EN VÍAS DE DESARROLLO EN EL MARCO DEL METABOLISMO URBANO: CASO CUENCA, ECUADOR. Universidad de jaen. Retrieved from http://ruja.ujaen. es/handle/10953/936

[30] [30]ARCONEL. (2019). Proyecto de Biogas Pichacay. Retrieved December 29, 2019, from https://www. regulacionelectrica.gob.ec/proyecto-de-biogas-pichacay/

[31] Urgiles, E., \& Yanez, I. (2019). Aplicación de Sistemas de Información Geográfica para la estimación del potencial energético de la biomasa lignocelulósica, en las áreas verdes públicas de la zona urbana de Cuenca. Universidad de Cuenca. Retrieved from http://dspace.ucuenca.edu.ec/handle/123456789/32119

[32] Izquierdo-torres, I. F., Pacheco-portilla, M. G., Gonzalez-Morales, L. G., \& Zalamea-Leon, E. F. (2019). Simulación fotovoltaica considerando parámetros de integración en edificaciones Photovoltaic simulation considering building integration parameters. INGENIUS:Revista de Cienciay Tecnología, 21,9-19.https://doi.org/https://doi.org/10.17163/ ings.n21.2019.02

[33] ARCH-Azuay, \& Centrosur. Base de datos (2017). Cuenca, Ecuador.

[34] Duffie, J. a., \& Beckman, W. a. (2013). Solar Engineering of Thermal Processes. Journal of Solar Energy Engineering (2nd ed., Vol. 116). New York. https://doi.org/10.1115/1.2930068

[35] International Energy Agency. (2019). Renewables Information 2019 Overview (Vol. 53). https://doi.org/10.1017/ CB09781107415324.004

[36] Castro Verdezoto, P. L., Vidoza, J. A., \& Gallo, W. L. R. (2019). Analysis and projection of energy consumption in Ecuador: Energy efficiency policies in the transportation sector. Energy Policy, 134(August). https://doi.org/10.1016/j. enpol.2019.110948

[37] Palacios, E., \& Espinoza, C. (2014). Contaminación Del Aire Exterior. Cuenca - Ecuador, 2009- 2013. Posibles Efectos En La Salud. Revista de Ciencias Médicas, 32(2), 34,56. Retrieved from http://dspace.ucuenca.edu.ec/ bitstream/123456789/22965/1/Dra Elvira palacios ing Claudia Espinoza.pdf

[38] González, L. G., Siavichay, E., \& Espinoza, J. L. (2019). Impact of EV fast charging stations on the power distribution network of a Latin American intermediate city. Renewable and Sustainable Energy Reviews, 107(June 2018), 309318. https://doi.org/10.1016/j.rser.2019.03.017 
[39] Zalamea-León, E., Mena-Campos, J., Barragán-Escandón, A., Parra-González, D., \& Méndez-Santos, P. (2018). URBAN PHOTOVOLTAIC POTENTIAL OF INCLINED ROOFING FOR BUILDINGS IN HERITAGE CENTERS IN EQUATORIAL AREAS. Journal of Green Building, 13(3), 45-69. https://doi.org/10.3992/1943-4618.13.3.45

[40] Karlessi, T., Kampelis, N., \& Kolokotsa, D. (2017). The concept of smart and NZEB buildings and the integrated design approach. Procedia Engineering, 180, 1316-1325. https://doi.org/10.1016/j.proeng.2017.04.294

[41] Marin-López, D., Zalamea-León, E. F., Barragán-Escandón, E. A., Marin-Lopez, D. S., Zalamea-León, E. F., \& BarragánEscandón, E. A. (2018). POTENCIAL FOTOVOLTAICO EN TECHUMBRE DE EDIFICIOS INDUSTRIALES DE ALTA DEMANDA ENERGÉTICA, EN ZONAS ECUATORIALES. A. Habitat Sustentable, 8(1), 28-41. https://doi.org/https://doi.org/10.223 20/07190700.2017.08.01.03 HS

[42] Zambrano-Asanza, S., Zalamea-León, E. F., Barragán-Escandón, A., Parra-González, D., Barragán-Escandón, E. A., \& Parra-González, A. (2019). Urban photovoltaic potential estimation based on architectural conditions, productiondemand matching, storage and the incorporation of new eco-efficient loads. Renewable Energy, 142, 224-238. https://doi.org/10.1016/j.renene.2019.03.105

[43] Ministerio Federal de Relaciones Exteriores de Alemania. (2017). La Energiewende Alemana.

[44] Freitas, S., Reinhart, C., \& Brito, M. C. (2018). Minimizing storage needs for large scale photovoltaics in the urban environment. Solar Energy, 159(September 2017), 375-389. https://doi.org/10.1016/j.solener.2017.11.011

[45] Bonomo, P., \& De Berardinis, P. (2014). PV integration in minor historical centers: Proposal of guidecriteria in postearthquake reconstruction planning. Energy Procedia, 48(0), 1549-1558. https://doi.org/10.1016/j.egypro.2014.02.175 